\title{
Thermal relaxation time and heat distribution in pulsed InGaAs quantum dot lasers
}

\author{
P. K. L. Chan ${ }^{\text {a) }}$ and K. P. Pipe \\ Department of Mechanical Engineering, University of Michigan, Ann Arbor, Michigan 48109-2125
}

Z. Mi, J. Yang, and P. Bhattacharya

Solid State Electronics Laboratory, Department of Electrical Engineering and Computer Science, University of Michigan, Ann Arbor, Michigan 48109-2122

D. Lüerßen ${ }^{\text {b) }}$

Research Laboratory of Electronics, Massachusetts Institute of Technology, Cambridge, Massachusetts 02139

(Received 18 December 2005; accepted 19 May 2006; published online 7 July 2006)

\begin{abstract}
Using a charge coupled device-based thermoreflectance technique, we achieve a high-resolution $(\sim 700 \mathrm{~nm})$ cross-sectional temperature profile of a semiconductor laser. This two-dimensional profile allows us to identify separate heat sources due to contact heating and nonradiative recombination in the active region. By adapting the technique to pulsed operation and varying the laser's duty cycle, we measure the thermal relaxation time constant. We also quantitatively determine the heat transfer from device-internal heat sources and demonstrate both the large effect of lateral heat spreading and the distinction between a laser's top surface temperature and its active region temperature. (C) 2006 American Institute of Physics. [DOI: 10.1063/1.2219721]
\end{abstract}

The performance and reliability of a semiconductor laser are strongly dependent on its operating temperature. ${ }^{1}$ However, the operating temperature is conventionally taken to be the target temperature of a controlled heat sink on which the laser is placed, rather than the temperature of the laser active region itself. This can lead to results which vary based on the thermal contact to the heat sink as well as on the heat flux capability of the heat sink; it has been shown that the majority of the temperature rise of a laser can actually occur within the heat sink for certain common heatsinking technologies due to their inefficiency at cooling microscale devices. ${ }^{2}$

In addition to deviation between the active region temperature and the target heat sink temperature, significant temperature variations can occur within the laser heterostructure itself. Large internal temperature gradients over submicron distances have been predicted for devices such as semiconductor lasers and transistors due to highly confined heat sources and low thermal conductivity layers. ${ }^{3}$ Thermoelectric effects at internal device heterojunctions ${ }^{4}$ and homojunctions ${ }^{5}$ have also been predicted which may work to create large device-internal temperature gradients.

In order to measure the temperature of active devices under bias, techniques such as IR thermography, photoluminescence, and Raman spectroscopy have previously been explored, although direct measurements of facet temperature in lasers have shown low spatial resolution. ${ }^{6-8}$ Furthermore, the latter two have been limited to a low temperature resolution $(\sim 10 \mathrm{~K})$ and require relatively intense pump light which can cause additional heating and affect accuracy. Single-spot thermoreflectance has good spatial, temperature, and time resolution $^{9,10}$ but proves difficult for generating a twodimensional (2D) map of device temperature. Such a 2D map can be important for understanding heat transfer mecha-

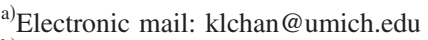

${ }^{b}$ Present address: Oxford Gene Technology, Oxford OX5 1PF, United Kingdom.
}

nisms within a device. For pulsed devices, heterodyned thermoreflectance techniques could be used for devices at high operating frequencies, ${ }^{11}$ but these techniques are not straightforward to apply at different duty cycles. The thermoreflectance technique described here offers a way to create a 2D map of temperature for a dc-biased device or a pulsed device at various duty cycles with high spatial and temperature resolution.

Thermoreflectance is a well-established temperature measurement technique for microscale electronic and optoelectronic devices due to its high resolution and relatively short experiment time. ${ }^{12}$ The basic idea is to measure the slight change $\Delta R$ in reflection coefficient $R$ of a sample surface caused by temperature variation $\Delta T$. The relationship is linear for small temperature variations and is given by $\Delta T$ $=\kappa^{-1} \Delta R / R$. The thermoreflectance coefficient $\kappa$ depends upon both the material and the illuminating wavelength, and has an order of magnitude $\sim 10^{-3}-10^{-5} \mathrm{~K}^{-1}$ for metals and semiconductors. It can be measured using thermocouples as part of a calibration procedure. ${ }^{12}$

Our experimental setup is shown in Fig. 1. A blue light emitting diode (LED) with $\lambda=470 \mathrm{~nm}$ is used to illuminate

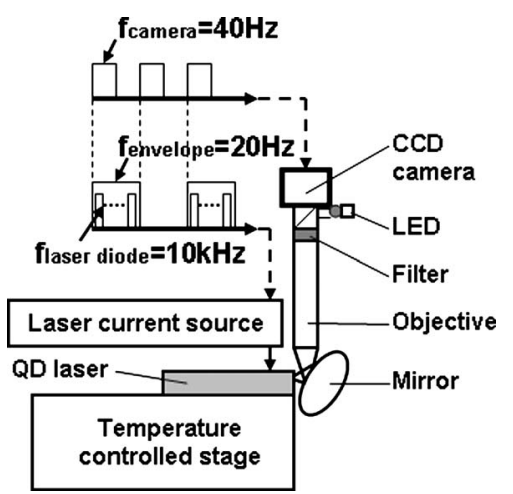

FIG. 1. CCD-based thermoreflectance setup for a pulsed laser diode. 


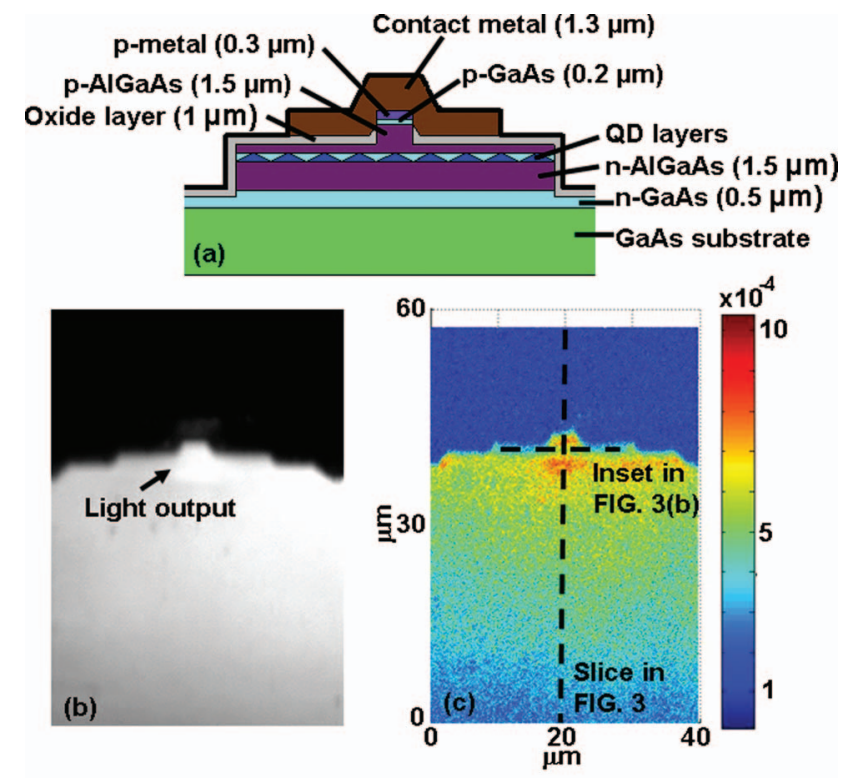

FIG. 2. (Color) Schematic view of the $\operatorname{In}(\mathrm{Ga}) \mathrm{As} / \mathrm{GaAs}$ quantum dot laser structure. (b) CCD image of the laser under bias without using a filter. A clear light output region can be seen at the laser active region. (c) Thermoreflectance $(\Delta R / R)$ image of the same device (with filter installed). The lower hot spot corresponds to the active region of the laser, and the upper hot spot corresponds to contact heating.

the laser through a microscope (using a $45^{\circ}$ mirror to image the laser facet), allowing us to have higher spatial resolution due to the smaller diffraction limit than techniques such as IR thermography. ${ }^{8}$ The LED light reflected backthrough the microscope objective $(100 \times$ with numerical aperature $=0.55)$ is imaged onto a 12-bit charge coupled device (CCD) camera. An OD-6 filter (Omega Optical 450DF100) is installed on the microscope to filter out the $(\lambda=1.1 \mu \mathrm{m})$ light generated by the laser under bias, and a temperaturecontrolled stage maintains the temperature under the laser at $20{ }^{\circ} \mathrm{C}$. We have confirmed experimentally that we cannot detect any laser light within the sensitivity levels used in our experiments when the filter is installed.

The camera is triggered on the rising edges of a $40 \mathrm{~Hz}$ signal, and the exposure (integration) time is set to $24.5 \mathrm{~ms}$. An envelope signal is created which is phase locked to the camera trigger signal with a frequency $20 \mathrm{~Hz}$ (half period $=25 \mathrm{~ms}$ ). Inside every other envelope half period, there are 250 cycles of a $10 \mathrm{kHz}$ signal used to drive the laser diode. The duty cycle of the laser can be adjusted by changing the pulse width setting of the laser diode driver; this does not, however, change the number of laser pulses per trigger pulse. In order to measure the very small variation in reflection between consecutive envelope half periods, we average the brightness value of each pixel over many periods $(10000$ $+)$. By setting the exposure time of the camera to be $24.5 \mathrm{~ms}$ ( $98 \%$ of the envelope half period), we maximize the integrated thermoreflectance signal. Note that by integrating we lose phase information in the signal.

The device under test is a $\lambda=1.1 \mu \mathrm{m} \operatorname{In}(\mathrm{Ga}) \mathrm{As} / \mathrm{GaAs}$ self-organized quantum dot laser on a GaAs substrate. ${ }^{13}$ The width of the ridge is $5 \mu \mathrm{m}$ and the length is $400 \mu \mathrm{m}$ [Fig. 2(a)]. An advantage of examining a quantum dot laser as opposed to a quantum well laser with this technique is that the measured facet temperature is likely to closely indicate the bulk temperature, since less facet heating is expected due
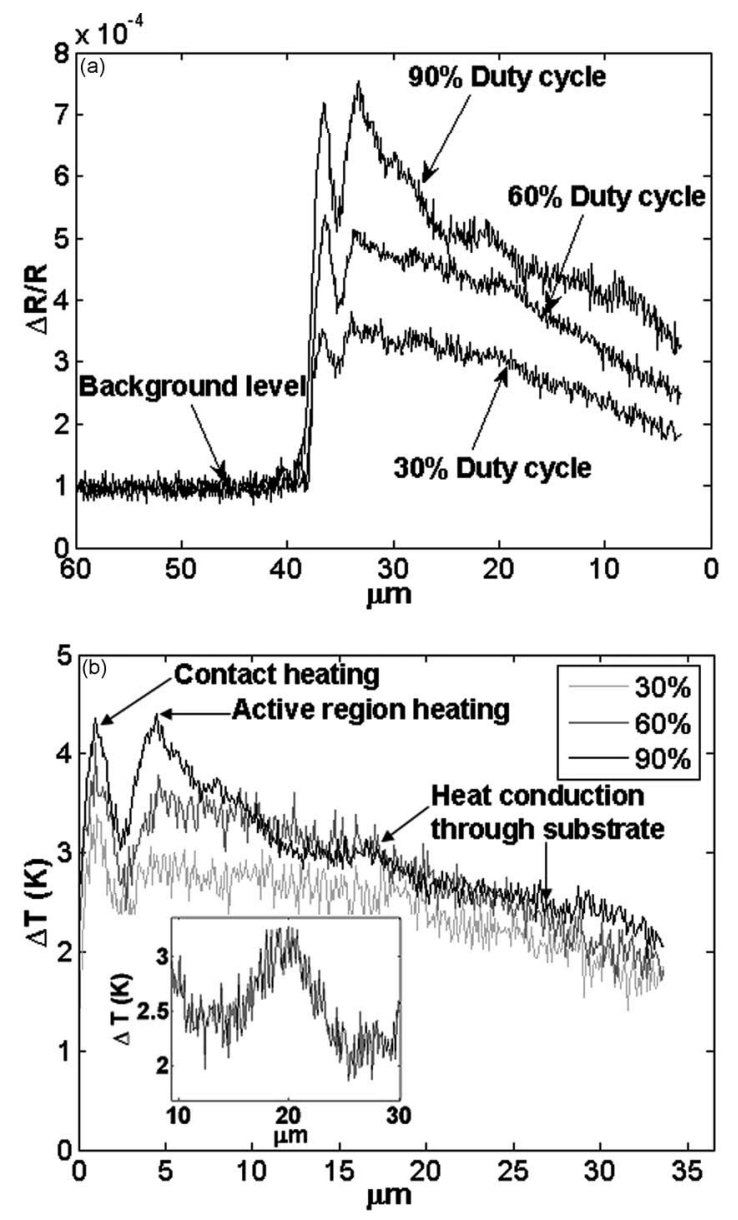

FIG. 3. Midplane thermoreflectance (a) and derived temperature (b) cross sections of the laser at $120 \mathrm{~mA}$ with different duty cycles. Strong lateral heat spreading leads to a dip in temperature between the heat sources at the top metal contact and active region. Inset: Lateral temperature profile across the laser ridge.

to reduced nonradiative recombination. ${ }^{14}$ A CCD image of the laser biased at $5 \mathrm{~mA}$ is shown in Fig. 2(b). In taking this image, we have removed the $\lambda=1.1 \mu \mathrm{m}$ filter, and the laser light output therefore appears on the CCD. We use both this image and our knowledge of the laser layer structure to locate the active region when examining the thermoreflectance data with the filter in place [shown in Fig. 2(c) for a bias of $120 \mathrm{~mA}$ and $90 \%$ duty cycle]. By overlaying Figs. 2(b) and 2(c), it is apparent that the lower "hot spot" in Fig. 2(b) is at the active region of the laser. From measuring the falloff in signal at the top edge of the laser, we derive a spatial resolution of $700 \mathrm{~nm}$. Figure 3(a) plots the thermoreflectance $(\Delta R / R)$ cross-section data at the slice illustrated in Fig. 2(c). In order to convert this $\Delta R / R$ data to $\Delta T_{\max }$ (the maximum temperature reached during pulsed operation), we must account for two factors: (1) the different values of $\kappa$ for different materials and (2) the time variation of $T$. Keeping the laser off and changing the entire sample temperature with the temperature-controlled stage, we compare $\Delta R / R$ with surface temperature microthermocouple measurements and find the value of $\kappa$ to be $1.9 \times 10^{-4} \mathrm{~K}^{-1}$ in the GaAs substrate and laser heterostructure and $1.8 \times 10^{-4} \mathrm{~K}^{-1}$ at the top of the laser ridge. These values agree with literature values for these materials. ${ }^{15} \mathrm{We}$ also run the experiment at the threshold current level $(20 \mathrm{~mA}, 1.4 \mathrm{~V})$ and detect no modulated reflectance signal; since this sweeps a large range of voltage and 


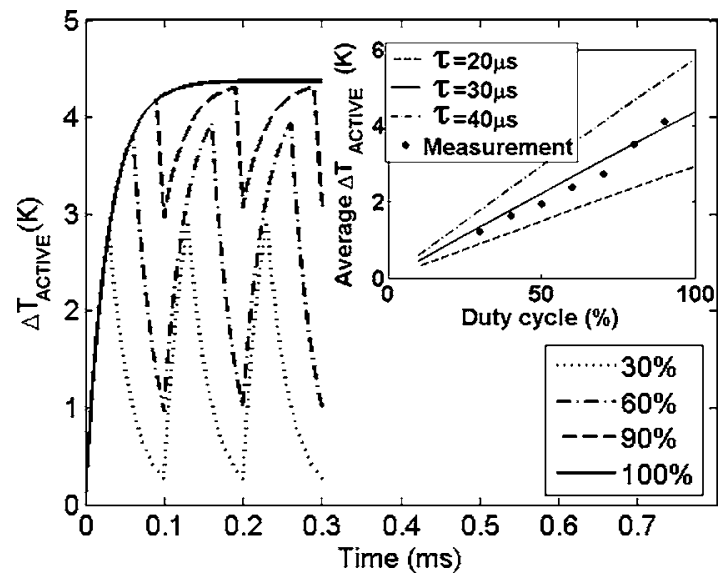

FIG. 4. Simulated active region temperature at $120 \mathrm{~mA}$ and different duty cycles for $\tau=30 \mu \mathrm{s}$. Inset: Simulated and measured active region temperature averaged over the CCD integration time $(24.5 \mathrm{~ms})$. Measurements indicate that the thermal time constant of the active region is $\sim 30 \mu \mathrm{s}$.

also because the laser active region carrier density is expected to clamp above threshold, we deduce that we can safely neglect electroreflectance in calibrating our measurement. $^{15}$

In order to obtain the maximum temperature profile during pulsed operation as shown in Fig. 3(b), a simple conversion factor (based on relating the maximum of an oscillating exponential function to its mean as shown in Fig. 4) has been used to relate the peak temperature value to the average temperature value. By fitting the results of a time-dependent finite-element simulation with the measured data at different duty cycles (both of which are averaged over the CCD integration time of $24.5 \mathrm{~ms}$ ), we find the thermal relaxation time constant $\tau$ of the laser active region to be approximately $30 \mu$ s (see Fig. 4 inset). Because laser performance depends strongly on temperature, this time constant governs the operation of the device.

From examining the standard deviation of the temperature data within the laser, we approximate the temperature resolution to be $200 \mathrm{mK}$. In Fig. 3(b) we can observe the continuous decay of temperature vertically from the active region through the substrate produced by heat transfer into the heat sink which is located below the laser. We can also see two separate heat sources within the laser, one at the top metal contact (which we associate with contact heating) and another at the QD active region (which we associate with nonradiative recombination). The temperature drop between the two laser heat sources suggests that a large fraction of the heat generated in both the contact region and the QD active region transfers laterally to the surface metal contact, which acts as a heat sink. The inset in Fig. 3(b) is the lateral temperature profile of the laser ridge between the two heat sources. A nearly symmetric temperature distribution can be observed, and the lateral temperature decay again indicates the lateral transfer of heat into the surface metal contact.

The thermal diffusivity $\alpha$ can be estimated by $\mu^{2} / \tau_{d}$ where $\mu$ is the thermal diffusion length and $\tau_{d}$ is the thermal diffusion time constant. By assuming $\tau_{d} \approx \tau$ and examining the decay of temperature laterally and vertically from the laser ridge as shown in Fig. 3(b) and its inset, we obtain an effective thermal diffusivity of $0.32 \mathrm{~cm}^{2} / \mathrm{s}$ for heat transfer vertically into the substrate (in agreement with literature values for GaAs), $0.26 \mathrm{~cm}^{2} / \mathrm{s}$ for heat transfer to the left into the metal contact, and $0.19 \mathrm{~cm}^{2} / \mathrm{s}$ for heat transfer to the right into the metal contact. Considering the temperature gradients and diffusivities in both directions, it is apparent that surface heat spreading and convection (usually ignored in laser heat transfer models) are important heat flow mechanisms for the device.

In summary, we performed CCD-based thermoreflectance imaging of a semiconductor laser in cross section, and also adapted the technique for pulsed devices. We generated a high-resolution 2D device temperature profile which allows us to see the internal temperature structure of a semiconductor laser from top contact to bottom contact, identifying separate internal heat sources due to contact heating and nonradiative recombination in the active region. By examining the device at different duty cycles, we derived the thermal time constant for the active region, and by examining the $2 \mathrm{D}$ temperature map, we derived the lateral and vertical thermal diffusivities from the laser ridge. This technique can be similarly applied to other electronic and optoelectronic devices (in pulsed or continuous-wave mode) to image internal temperature profiles.

The work is supported by the Defense Advanced Research Projects Agency through the EPIC program under Grant No. W911NF-04-1-0429.

${ }^{1} \mathrm{M}$. Fukuda, Reliability and Degration of Semiconductor Lasers and LEDS (Artech, Boston, 1991).

${ }^{2}$ K. P. Pipe and R. J. Ram, IEEE Photonics Technol. Lett. 15, 504 (2003).

${ }^{3}$ S. Sinha and K. E. Goodson, THERMINIC 2004, Sophia Antipolis, France.

${ }^{4}$ K. P. Pipe, R. J. Ram, and A. Shakouri, IEEE Photonics Technol. Lett. 14, 453 (2002).

${ }^{5}$ H. K. Lyeo, A. A. Khajetoorians, L. Shi, K. P. Pipe, R. J. Ram, A. Shakouri, and C. K. Shih, Science 303, 816 (2004).

${ }^{6}$ S. J. Sweeney, L. J. Lyons, A. R. Adams, and D. A. Lock, IEEE J. Sel. Top. Quantum Electron. 9, 1325 (2003).

${ }^{7}$ W. C. Tang, H. J. Rosen, P. Vettiger, and D. J. Webb, Appl. Phys. Lett. 58, 557 (1991).

${ }^{8}$ A. Kozlowska, M. Latoszek, J. W. Tomm, F. Weik, T. Elsaesser, M. Zbroszczyk, M. Bugajski, B. Spellenberg, and M. Bassler, Appl. Phys. Lett. 86, 203503 (2005).

${ }^{9}$ S. Dilhaire, S. Grauby, S. Jorez, LD. Lopez, E. Schaub, and W. Claeys, Microelectron. Reliab. 41, 1597 (2001).

${ }^{10}$ T. Hayakawa, Appl. Phys. Lett. 75, 1467 (1999).

${ }^{11}$ S. Grauby, B. C. Forgét, S. Holé, and D. Fournier, Rev. Sci. Instrum. 70, 3603 (1999).

${ }^{12}$ D. Lüerßen, J. A. Hudgings, P. M. Mayer, and R. J. Ram, IEEE 21st Semi-therm Symposium, 2005, p. 253.

${ }^{13}$ S. Fathpour, Z. Mi, and P. Bhattacharya, IEEE Photonics Technol. Lett. 17, 2250 (2005).

${ }^{14}$ J. W. Tomm, F. Rinner, J. Rogg, E. Thamm, C. Ribbat, R. Selling, and D. Bimberg, Proc. SPIE 4993, 91 (2003).

${ }^{15}$ P. W. Epperlein, Jpn. J. Appl. Phys., Part 1 32, 5514 (1993). 